\title{
The Sequential Indeterminacy Problem
}

\author{
[Forthcoming in \\ General equilibrium: problems, prospects and alternatives \\ Frank Hahn and Fabio Petri, eds.]
}

\author{
Michael Mandler \\ Department of Economics \\ Royal Holloway College \\ University of London \\ Egham, Surrey TW20 0EX \\ United Kingdom
}

August 1999

\begin{abstract}
:
General equilibrium models generically are determinate and have a finite number of equilibria. But, with linear activities and some inelastically supplied factors of production, the economies that arise endogenously as perfect-foresight equilibria proceed through time can robustly be indeterminate. During the initial period of an intertemporal model, the perfect-foresight equilibria typically are well-behaved, but they can generate later-period endowments for agents such that the equilibria that validate perfect-foresight expectations lie amid a continuum of other equilibria. Since later-period equilibria are not continuous functions of endowments and other parameters, agents (even if small relative to the market) have an incentive to manipulate market prices; the assumption that agents are price takers therefore breaks down. Sequential indeterminacy is related to Sraffa's indeterminacy results in that (1) smooth "neoclassical" technologies eliminate indeterminacy, and (2) the dimension of sequential indeterminacy matches the dimension of indeterminacy in Sraffa's model. Despite the link to Sraffa, long-run equilibria, where relative prices are constant through time, are generically determinate.
\end{abstract}




\section{Introduction}

Factor pricing was once one of the most contentious subjects in economics. From the late 19th through the mid 20th century, champions and critics of marginal productivity argued forcefully about the nature of technology and whether the infinite array of the factor substitution possibilities implied by the differentiable, "neoclassical" production function is realistic. Although the neoclassical production function remains the most common model of production, arguments about the nature of technology seem puzzling today: Arrow-Debreu general equilibrium theory does not impose differentiability assumptions on production functions or production sets. Premodern theorists thus seem to have been debating an irrelevancy.

I argue that earlier worries about factor substitution were well-justified. In the absence of sufficient substitutability, factor demand can be inelastic. If factor supply is also inelastic, equilibrium factor prices will not be determinate. Indeterminacy is not just a technical nuisance; it undermines the price-taking assumption of competitive equilibrium theory. In the indeterminacy under study, arbitrarily small reductions in factor supplies can discontinuously increase a factor's price, and consequently factor owners will not take prices to be parametric.

Standing opposed to the possibility of indeterminacy, the regularity literature of general equilibrium theory shows that competitive equilibria generically are determinate; that is, for almost every configuration of parameters, general equilibrium models have only locally unique equilibria. Since this result holds for models of production with limited or even no possibilities for factor substitution and when factors are inelastically supplied, the regularity literature implicitly contends that factor price indeterminacy is not an important problem.

I show, however, that in intertemporal models in which agents trade at multiple dates (instead of once-and-for-all at the beginning of economic time), it can occur robustly that the equilibrium behavior of agents endogenously generates the parameters at which indeterminacy occurs. At the start of an intertemporal model, the perfect-foresight equilibria generically are 
determinate, but those equilibria can generate endowments for agents such that in later periods the equilibrium that validates the perfect-foresight expectations is contained within a continuum of other equilibria. This is the sequential indeterminacy problem. Although at each period $t$ the equilibria of the economy that begins at $t$ would generically be determinate if we could perturb endowments at $t$ and later, economies are driven over time to precisely the measure-zero set of endowments that cause trouble.

I begin by reviewing the standard determinacy theorems of the regularity literature, both in general and in a two-factor example, and then show how an intertemporal equilibrium can be decomposed into an equilibrium with sequential trading. For simplicity, our sequential trading equilibria involve trading at just two dates. The decomposition of each intertemporal equilibrium defines a set of economies that operate during the second time period. It can turn out that almost all of these economies have indeterminate equilibria. Technologies with limited factor-substitution possibilities, such as linear activities, are indispensable to sequential indeterminacy; as we will see, the perfect-foresight equilibria of models with differentiable neoclassical technologies typically are determinate during their later periods of operation. The importance of the differentiability of technology hints at a link to Sraffa's famous indeterminacy claims. The connection turns out to be tight, except for one important proviso: sequential indeterminacy will not arise in the favored Sraffian environment of long-run equilibria.

2. Determinacy in general equilibrium theory

Despite the considerable attention theorists paid to determinacy prior to World War II, postwar general equilibrium theory initially ignored the subject. But after 1970, when Gerard Debreu published his path-breaking article on the number of equilibria in exchange economies, the modern literature developed rapidly. For models with a finite horizon (and as long as assets are real, not financial), the regularity literature has yielded remarkably sharp conclusions. 
Typical, or generic, models have a finite number of equilibria. And these equilibria have appealing comparative statics properties: equilibrium prices and allocations change smoothly as a function of the parameters of the model. Consequently, in large economies, agents can have only a small effect on equilibrium prices and therefore have little incentive to manipulate markets. For example, as the number of agents becomes large, the effect on equilibrium prices of an agent withholding a portion of his or her endowment from the market becomes small. In the limit, agents take prices to be parametric and act competitively (Roberts (1980)). The study of determinacy thus not only resolved a technical issue - whether the number of equilibria is finite or infinite - but also shored up the foundational story of when markets operate competitively.

For production economies, Mas-Colell (1975) and Kehoe (1980, 1982) established generic determinacy results for constant-returns-to-scale technologies and for the linear activity analysis model in particular. ${ }^{1}$ From the perspective of the history of production theory, the MasColell-Kehoe results are remarkable. The original popularity of the differentiable production function, after all, was due to the fact that it ensures that factor demand is elastic with respect to price; hence, even if factor supply is inelastic, factor price indeterminacy will not occur. But although differentiability secured this important theoretical goal, it was also attacked by many as empirically unrealistic (see Mandler (1999a) for a more detailed history). The modern regularity literature, in contrast, seems to show that earlier assertions of indeterminacy had been incorrect: determinacy is generic with virtually any description of technology and whether or not factors are inelastically supplied. The decades-long debate between the "fixed coefficients" and "differentiable production function" camps thus appears to have been pointless.

A model in the spirit of Mas-Colell and Kehoe will serve as our reference point. We

1 Mas-Colell and Kehoe's work was preceded by Fuchs (1974) and Smale (1974), which analyzed decreasing-returns-to-scale technologies. Mas-Colell (1985) provides a definitive overview of the regularity literature. 
assume there are $L$ goods that provide agents with utility and $M$ goods that agents supply inelastically. The $L$ goods may be either pure consumption goods or factors, such as labor time, whose demand varies as a function of prices, while the $M$ goods are pure factors of production. We suppose there are $N$ activities, $a_{1}, \ldots, a_{N}$, each $a_{j} \in R^{L^{+M}}$ indicating the quantities of the $L+$ $M$ goods necessary to run activity $j$ at the unit level, and summarized by the $L+M$ by $N$ technology matrix $A=\left[\begin{array}{lll}a_{1} \cdots & a_{N}\end{array}\right]$. The $i$ th row-j th column element of $A, a_{i j}$, is the quantity of good $i$ used (if $a_{i j}<0$ ) or produced (if $a_{i j}>0$ ) by activity $j$. Letting $y \in R_{+}^{N}$ indicate the vector of activity or usage levels, aggregate production equals $A y$. The production set that arises from this technology is $Y=\left\{\gamma \in R^{L+M}: \exists y \in R_{+}^{N}\right.$ s.t. $\left.\gamma \leq A y\right\}$. We make the standard assumption that production of positive amounts of all goods is impossible: $Y$ intersects the positive orthant only at $\{0\}$.

We assume there are a finite number of agents. A typical agent $k$ is described by (1) a twice continuously differentiable utility function $u^{k}: R_{+}^{L} \rightarrow R$ that is differentiably increasing and differentiably strictly concave and where no indifference surface through any $x^{k} \in R_{++}^{L}$ intersects any coordinate axis, and (2) an endowment $\left(e_{c}^{k}, e_{f}^{k}\right) \in R_{++}^{L+M}$ of goods and factors. ${ }^{2}$ These assumptions can be weakened considerably. In particular, agents could be endowed only with natural resources, and not with producible goods as well.

Let $(p, w) \in R_{+}^{L+M} \backslash\{0\}$ denote the prices of goods and factors. Each agent $k$ 's budget set is then $\left\{x^{k} \in R_{+}^{L}: p \cdot x^{k} \leq w \cdot e_{f}^{k}+p \cdot e_{c}^{k}\right\}$. Maximizing $u^{k}$ subject to this budget set generates the excess demand function $z^{k}(p, w)$, and, summing, the aggregate excess demand $z(p, w)=$ $\sum_{k} z^{k}(p, w)$. The excess demand for the $M$ pure factors is simply $-e_{f}=-\sum_{k} e_{f}^{k}$. The aggregate excess demand function for the $L+M$ goods taken together is therefore $\left(z(p, w),-e_{f}\right)$. Let $e_{f}(i)$ denote the aggregate endowment of factor $i$.

2 "Differentiably increasing" and "differentiably strictly concave" mean that, for all $x^{k} \in$ $R_{++}^{L}, D u^{k}\left(x^{k}\right) \gg 0$ and $D^{2} u^{k}\left(x^{k}\right)$ is negative definite. 
An equilibrium is a $(p, w, y)$ such that $\left(z(p, w),-e_{f}\right) \leq A y$ and $(p, w)^{\prime} A \leq 0$. Using Walras' law, it is easy to confirm that if any of the market-clearing inequalities is strict, then the corresponding price equals zero, and that if any of the no-positive-profits inequalities is strict, then the corresponding coordinate of $y$ equals 0 . Under our assumptions, an equilibrium exists.

The regularity literature takes the view that properties of a model that obtain only at exceptional, "nongeneric" combinations of parameters can be dismissed as unlikely. This principle should not be applied naively, but, if modified so as to not rule out seemingly unlikely parameters configurations that in fact arise endogenously, the underlying precept is sound. Indeed, in order to show that a property arises systematically in the later periods of an intertemporal model, one must ensure that it arises for a nonnegligible set of intertemporal economies. So, whether from the regularity literature's vantage point or from a sequential point of view, an explicit space of economies or parameters is indispensable.

We now specify our parameter space and define a "regular" economy. Roughly speaking, the key feature of a regular economy is that each vector of equilibrium relative prices and activity levels is locally unique and varies differentiably as function of the economy's parameters. The pertinent equilibrium variables of a regular economy can in fact be viewed as the solution of a simple system of equations: the market-clearing conditions for all the goods not in excess supply (less one, due to Walras' law) and the zero-profit conditions of activities that make exactly zero profits. Regular economies, moreover, comprise a generic subset of the space of economies. The exact concepts needed to define regularity are a little involved; the reader may wish to proceed to the example discussed in the next section.

The parameter space will be the cross product of the open set of endowments that meet the assumptions imposed above and an open set of technology matrices to be defined momentarily. Since technology matrices and endowments can each be identified with finitedimensional vectors of real numbers, the concepts of openness, density, and full-measure sets 
will have their familiar Euclidean meanings. We must take a little care with technology matrices. If each entry in a $L+M$ by $N$ technology matrix is a free parameter, then almost every technology matrix will specify that each good is either an input or an output in each activity. This would be absurd in any model, and physically impossible once we distinguish goods by the date at which they appear. Therefore, given an arbitrary, fixed technology matrix $A$ meeting our assumptions, let $A^{\prime}$ be admissible if, for all $i$ and $j, \operatorname{sgn}\left(a_{i j}{ }^{\prime}\right)=\operatorname{sgn}\left(a_{i j}\right)$. Our parameter space for technology matrices will be the set of admissible matrices. A generic set is a subset of our space of economies that is open and whose complement has measure zero. ${ }^{3}$

To analyze equilibria (locally) as the solution of a system of equations rather than inequalities, consider the subset of the conditions $\left(z(p, w),-e_{f}\right) \leq A y$ and $(p, w)^{\prime} A \leq 0$ that "bind" at some equilibrium $(p, w, y)^{\star}$ : the market-clearing conditions where demand exactly equals supply and the no-positive-profits conditions for those activities that both exactly break even and utilize or produce at least one good not in excess supply. All of the market-clearing conditions for consumption goods are included in this subset, but some factors may be in excess supply and some (unused) activities may make strictly negative profits or utilize/produce only factors in excess supply. For $(p, w, y)$ near $(p, w, y)^{\star}$, the excluded factors will continue to be in excess supply and the excluded activities continue either to make strictly negative profits or to utilize/produce only factors in excess supply. Consequently, in a neighborhood of $(p, w, y)^{\star}$, we may ignore these excluded equilibrium conditions: they are automatically satisfied. In addition,

${ }^{3}$ For the Sequential Indeterminacy Theorem of section 6, we will need to supplement the basic parameter space with a space of utility functions. Given a utility $u^{k}$ for agent $k$ that meets the assumptions stated above, let $u^{k}+\varepsilon f$ be an admissible utility for $k$, where $f$ is any quadratic function on $R_{+}^{L}$ and where $\varepsilon$ is a scalar in an open set small enough that $u^{k}+\varepsilon f$ satisfies the same assumptions when restricted to the set of feasible consumption vectors that deliver at least as much utility for $k$ as $e_{c}{ }^{k}$. Since quadratic functions can be parameterized by a finite set of real numbers, our space of economies can still be seen as an open set of finite-dimensional vectors of real numbers. 
we may use Walras' law, which implies that if all but one of the economy's market-clearing equalities is satisfied then so is the remaining one, to eliminate one of the equalities in $\left(z(p, w),-e_{f}\right) \leq A y$. We choose to omit the first consumption good. Finally, given the homogeneity of $z(p, w)$ and $(p, w)^{\prime} A$, we may set the price of one of the consumption goods say the first - equal to 1 without restricting the set of equilibrium allocations.

To specify our new system of equations and unknowns explicitly, we define $\bar{p}$ by setting the first coordinate of $p$ equal to $1, \bar{w}$ and $\bar{e}_{f}$ by omitting the coordinates of $w$ and $e_{f}$ that correspond to the factors in excess supply, $\bar{z}(\bar{p}, \bar{w})$ by omitting the first coordinate of the range of $z, \bar{A}$ by omitting the rows of $A$ that correspond to the factors in excess supply and the columns that correspond to the negative-profit activities or activities that utilize/produce only factors in excess supply, $\bar{y}$ by omitting the same activity level variables, and, finally, $\tilde{A}$ by omitting the row of $\bar{A}$ that corresponds to the first consumption good.

Keep in mind that these definitions are assembled relative to a particular equilibrium: depending on which factors are in excess supply and which activities break even, a different set of equilibrium conditions will bind. We will now typically use equilibrium to refer to a $(\bar{p}, \bar{w}, \bar{y})$. To say that $(\bar{p}, \bar{w}, \bar{y})$ is an equilibrium means simply that there exists an equilibrium in our previous sense where the prices omitted from $(\bar{p}, \bar{w})$ are set to 0 and all money-losing activities omitted from $\bar{y}$ have usage levels equal to 0 .

Given an equilibrium $(\bar{p}, \bar{w}, \bar{y})$, equilibria near $(\bar{p}, \bar{w}, \bar{y})$ are characterized by the equations $\left(\bar{z}(\bar{p}, \bar{w}),-\bar{e}_{f}\right)=\tilde{A} \bar{y}$ and $(\bar{p}, \bar{w})^{\prime} \bar{A}=0$. These equations contain as many equations as there are variables in $(\bar{p}, \bar{w}, \bar{y})$. The basic requirement of regularity is that this system of equations has "full rank," which will imply the local uniqueness of $(\bar{p}, \bar{w}, \bar{y})$. To this end, define $\left.F(\bar{p}, \bar{w}, \bar{y})=\left(\left(\bar{z}(\bar{p}, \bar{w}),-\bar{e}_{f}\right)-\tilde{A} \bar{y}\right),(\bar{p}, \bar{w})^{\prime} \bar{A}\right)$. An equilibrium is regular if (1) $D F$, evaluated at $(\bar{p}, \bar{w}, \bar{y})$, is nonsingular (i.e., has rank equal to $L-1$ plus the number of factors in $\bar{w}$ plus the number of activities in $\bar{y}$ ), (2) each activity $j$ whose zero-profit condition is exactly 
satisfied has $y(j)>0$, and (3) each factor $i$ not in excess supply has $w(i)>0$.

If an equilibrium is regular, equilibrium prices and quantities are locally a differentiable function of our parameterized set of economies. To see this, consider an economy $\left(e^{*}, A^{*}\right)$ with a regular equilibrium $\left(\bar{p}^{*}, \bar{w}^{*}, \bar{y}^{*}\right)$ and adjoin the parameter space to the domain of $F$. The implicit function theorem implies there is a continuously differentiable function of the parameters in a neighborhood of $\left(e^{*}, A^{*}\right)$ that identifies the unique $(\bar{p}, \bar{w}, \bar{y})$ in a neighborhood of $\left(\bar{p}^{*}, \bar{w}^{*}, \bar{y}^{*}\right)$ that satisfies $F(\bar{p}, \bar{w}, \bar{y})=0$. In a small enough neighborhood, these $(\bar{p}, \bar{w}, \bar{y})$ must indeed be equilibria: (i) by holding fixed the usage levels of any activity that utilizes/produces only factors in excess supply at $\left(\bar{p}^{*}, \bar{w}^{*}, \bar{y}^{*}\right)$, any market-clearing condition omitted from the range of $F$ must still be satisfied, (ii) any activity's profit level that is omitted from the range of $F$ and that makes negative profits at $\left(\bar{p}^{*}, \bar{w}^{*}, \bar{y}^{*}\right)$ will continue to make negative profits, (iii) any activity that utilizes/produces only factors in excess supply at $\left(\bar{p}^{*}, \bar{w}^{*}, \bar{y}^{*}\right)$ will plainly continue to break even, and (iv) given (2) and (3), ( $\left.\bar{p}, \bar{w}, \bar{y}\right)$ will be nonnegative. Since $F(\bar{p}, \bar{w}, \bar{y})=0$ has a locally unique solution for the parameters $\left(e^{*}, A^{*}\right)$, any regular equilibrium is locally isolated. ${ }^{4}$

An economy is defined to be regular if all of its equilibria are regular. Under our assumptions, it can additionally be shown that a regular economy has a finite number of equilibria.

Generic Determinacy Theorem (Mas-Colell/Kehoe). The regular economies form a generic set.

We have taken care to include in the model goods that do not give agents utility. The

4 The usage levels for any activities that utilize or produce only goods that are in excess supply will be indeterminate. This fact leads to no corresponding indeterminacy of relative prices or agents' welfare, however. 
above theorem therefore establishes generic determinacy in the important case where technology is described by linear activities and factors are inelastically supplied.

3. Regularity and determinacy in a two-factor example

To see an example of how different parameter configurations can generate regular economies or the troublesome cases that cause indeterminacy, let there be one consumption good and two factors ( $L=1, M=2)$, suppose that each activity produces only the consumption good, and normalize the activities so that the unit level of each activity produces one unit of that good. Label the pure factors so that they have commodity indices 1 and 2. Setting the price of the single consumption good equal to 1 , and using Walras' law to ignore the market-clearing condition for the consumption good, equilibria are characterized by:

$$
\begin{array}{ll}
-\sum_{j} a_{i j} y_{j} \leq e_{f}(i), & i=1,2, \\
1+w(1) a_{1 j}+w(2) a_{2 j} \leq 0, & j=1, \ldots, N .
\end{array}
$$

Due to the sign convention, each $a_{i j}$ in (3.1) and (3.2) is negative.

For generic technology matrices, at most two activities are used in equilibrium and all unused activities will satisfy (3.2) with strict inequality. When exactly two activities are in use, the corresponding two equalities in (3.2) determine unique values for $w(1)$ and $w(2)$, and for generic technologies both of these numbers will be strictly positive in equilibrium. Thus, in this regular case, neither factor can be in excess supply. The usage levels of the two activities are determined by (3.1), both of which are equalities. At equilibria where only one activity is in use, on the other hand, there is only one equality in (3.2) to determine both $w(1)$ and $w(2)$. If one of the factors is in excess supply, its price must equal zero and regularity and determinacy still obtain. But if both factor market-clearing conditions are satisfied with equality (and thus neither factor price is constrained to equal 0), and if, as with regular equilibria, the inequalities in (3.2) for the unused activities are strict, the equilibrium is indeterminate. A continuum of values for 
$w(1)$ and $w(2)$ will obey the one equality in (3.2) while still satisfying the strict inequalities in (3.2). And since no prices appear in (3.1), any of these $(w(1), w(2))$ can serve as equilibrium factor prices.

Summing up, indeterminacy occurs if neither factor is in excess supply and just one activity is in use, while prices are determinate (for generic activity analysis matrices) if two activities are in use or if one of the factors is in excess supply.

The Generic Determinacy Theorem therefore implies that equilibria with just one activity in use but with two fully employed factors occur only at a zero-measure set of economies. This fact is easy to confirm directly. With neither factor in excess supply, (3.1) contains exactly two equalities. But since only one activity is in use, only one of the components of $y$, say $y_{j}$, is nonzero. Except for a zero-measure set of the endowment vectors $e_{f}$, therefore, there exists no value for $y_{j}$ that satisfies the two equalities in (3.1). Hence, generically, indeterminacy does not occur. The regular, determinate equilibria, in contrast, arise robustly and vary differentiably as a function of $e_{f}$. For example, when two activities are in use, (3.1) contains two equalities and there are two nonzero activity levels; for generic activity analysis matrices, the equalities in (3.1) in this case have a unique solution for every $e_{f}$.

Figure 1 graphs the set of possible endowment points. The Figure supposes that there are two activities, each represented by the ray of factor combinations that the activity can fully employ. The interiors of the three cones of endowments lead to determinacy: in the interior of the two outside cones, one activity is in use and one of the two factors is in excess supply, while in the interior of the inner cone, two activities are in use and both factors are fully employed. Only at the lower-dimensional set of endowments that lie on the two rays, where only one activity is in use and both factors are fully utilized, does indeterminacy occur.

[Insert Figure 1]

Two representative isoquants, which links points on the activity rays that produce the 
same quantity of the consumption good, are also pictured. Along the activity rays, where indeterminacy occurs, a continuum of price lines support the isoquant; marginal products for the factors consequently are not well-defined. In the cone interiors, in contrast, only a single price line supports the isoquant and marginal products are defined. The relationship between determinacy and marginal productivity generalizes: with arbitrary numbers of activities and factors and a single consumption good, marginal products are well-defined at the same generic set of factor endowments at which equilibria are locally unique. Thus, generically at least, marginal productivity theory does not require that differentiable production functions are posited as a primitive; linear activities can serve as their foundation.

\section{Determinacy vs. sequential determinacy}

The standard Arrow-Debreu model supposes that agents meet once and contract for delivery of goods at all moments of economic time. The determinacy that the regularity literature establishes, therefore, is the determinacy of equilibria for these sorts of markets. The standard literature simply does not address whether the economies that endogenously emerge through time have determinate or indeterminate equilibria.

As an intertemporal economy proceeds through time, the endowments of agents, and other parameters, endogenously evolve. The endowments that generate indeterminacy in one of the economy's later time periods, even though they form a measure-zero subset of the endowment space of that period, might therefore arise systematically. This outcome is more than an abstract possibility; optimizing agents accumulate capital goods only in specific configurations. We will see that in equilibrium the production of capital goods can generate precisely the endowments that lead to indeterminacy. 
5. Decomposing an intertemporal equilibrium into a sequence of markets

The simplest way to model an economy proceeding through time is to define the intertemporal equilibria, where agents trade at only one date, and then reinterpret those equilibria as occurring via trading at a sequence of dates. See Radner (1972) for a general theory of such reinterpretations or "decompositions." To generate sequential indeterminacy, it will suffice to consider models with trading at just two periods. But each date might represent a multi-period composite, with the two dates forming a partition of a larger underlying set of time periods.

We begin by rewriting the model of section 2 as a two-period intertemporal model. In the second period, there are $L_{2}$ consumption goods, $M_{2}$ pure factors, and $N_{2}$ activities that commence within the period. There are $N_{1}$ activities that commence within the first period, and $L_{1}$ firstperiod goods. The first-period goods could be a mixture of consumption goods and pure factors, but to minimize notation we assume they are all consumption goods. The intertemporal activity analysis matrix has the form

$$
A=\left(\begin{array}{cc}
A_{11} & 0 \\
0 & A_{22} \\
A_{31} & A_{32}
\end{array}\right) \cdot \begin{gathered}
L_{1} \\
L_{2} \\
M_{2}
\end{gathered}
$$

The second subscript of each $A_{i j}$ indicates the period during which the activity commences. The 0 submatrices in $A$ indicate that we are assuming, again for notational simplicity, that activities in each period do not directly produce or utilize consumption goods that belong to the other period.

Each agent $k$ of the intertemporal model is described by a utility function $u^{k}\left(x_{1}{ }^{k}, x_{2}^{k}\right)$, where $\left(x_{1}^{k}, x_{2}^{k}\right)$ is $k$ 's first- and second-period consumption and by endowments $\left(e_{c_{1}}^{k}, e_{c_{2}}^{k}, e_{f_{2}}^{k}\right)$ of first-period goods, second period consumption goods, and second-period factors.

The primitives of the intertemporal model - the utility functions, the endowment vectors, and the technology matrix - are merely two-period versions of the primitives of the model of section 2, and we assume that the current primitives obey the assumptions imposed there. An 
intertemporal equilibrium, which is a $\left(p_{1}, p_{2}, w_{2}, y_{1}, y_{2}\right)$, therefore always exists, and the Generic Determinacy Theorem applies. Throughout the remainder of this paper, we consider only regular two-period economies. This restriction ensures both that second-period parameters are determined endogenously and that those parameters are not unlikely from the perspective of the overall two-period model.

We now reinterpret the intertemporal equilibria as equilibria with sequential trading and perfect foresight. In order that the two time periods are properly linked, agents must be able to purchase or sell assets in period 1 for delivery during period 2. We assume that the assets in the model consist of those second-period pure factors that are outputs of some first-period activity and that there are $M_{\alpha}>0$ such factors. These goods will be indexed as the first $M_{\alpha}$ of the second-period factors. Let $\alpha^{k}$ represent agent $k$ 's purchases of assets; $\alpha^{k}$ will be an element of $R^{M_{2}}$ but the last $M_{2}-M_{\alpha}$ coordinates of $\alpha^{k}$ are constrained to equal 0 . Similarly, $q \in R^{M_{2}}$ will have the prices of the assets as its first $M_{\alpha}$ coordinates and will equal 0 elsewhere. Let $p_{1}$ denote the prices of the first-period goods, $p_{2}$ the prices of the second-period consumption goods, and $w_{2}$ the prices of the second-period factors.

Each agent $k$ maximizes $u^{k}\left(x_{1}^{k}, x_{2}^{k}\right)$ subject to the budget constraints

$$
p_{1} \cdot x_{1}^{k}+q \cdot \alpha^{k} \leq p_{1} \cdot e_{c_{1}}^{k} \text { and } p_{2} \cdot x_{2}^{k} \leq w_{2} \cdot\left(\alpha^{k}+e_{f_{2}}^{k}\right)+p_{2} \cdot e_{c_{2}}^{k} \text {. }
$$

In order for agents to be willing to hold all $M_{\alpha}$ assets, the first $M_{\alpha}$ coordinates of $w_{2}$ must be proportional to the same coordinates of $q$ : hence in equilibrium there must exist a $R>0$ such that, for $i=1, \ldots, M_{\alpha}, R q(i)=w_{2}(i)$. Using this substitution, agent $k$ 's budget constraints reduce to

$$
p_{1} \cdot x_{1}^{k}+\left(\frac{1}{R}\right) p_{2} \cdot x_{2}^{k} \leq p_{1} \cdot e_{c_{1}}^{k}+\left(\frac{1}{R}\right) w_{2} \cdot e_{f_{2}}^{k}+\left(\frac{1}{R}\right) p_{2} \cdot e_{c_{2}}^{k}
$$

Utility maximization subject to this constraint generates the excess demand functions $z_{1}^{k}\left(p_{1}, p_{2}, w_{2}, R\right)$ and $z_{2}^{k}\left(p_{1}, p_{2}, w_{2}, R\right)$ for the first and second period consumption, and, 
summing, the aggregate excess demand functions $z_{1}\left(p_{1}, p_{2}, w_{2}, R\right)$ and $z_{2}\left(p_{1}, p_{2}, w_{2}, R\right)$.

Letting $y_{1}$ and $y_{2}$ denote the activity levels for the two periods and defining $e_{f_{2}}=\sum_{k} e_{f_{2}}^{k}$, the market clearing requirements appear as

$$
\begin{aligned}
& z_{1}\left(p_{1}, p_{2}, w_{2}, R\right) \leq A_{11} y_{1}, \\
& -A_{32} y_{2} \leq A_{31} y_{1}+e_{f_{2}}, \\
& z_{2}\left(p_{1}, p_{2}, w_{2}, R\right) \leq A_{22} y_{2},
\end{aligned}
$$

while the zero-profit conditions take the form

$$
p_{1}{ }^{\prime} A_{11}+\left(\frac{1}{R}\right) w_{2}{ }^{\prime} A_{31} \leq 0 \text { and } p_{2}{ }^{\prime} A_{22}+w_{2}{ }^{\prime} A_{32} \leq 0 \text {. }
$$

A sequential trading equilibrium is a $\left(p_{1}, p_{2}, w_{2}, R, y_{1}, y_{2}\right)$ that satisfies (5.1) and (5.2). It is immediate that $\left(p_{1}, p_{2}, w_{2}, R, y_{1}, y_{2}\right)$ is a sequential trading equilibrium if and only if $\left(p_{1},(1 / R) p_{2},(1 / R) w_{2}, y_{1}, y_{2}\right)$ is an intertemporal equilibrium. Indeed, the only difference between the models is that in the sequential model agents make asset choices and face two budget constraints. But since each agent's reduced-form budget constraint replicates the budget constraint of the intertemporal model, the two equilibrium concepts coincide. ${ }^{5}$

In defining a sequential trading equilibrium, we did not need to specify the $\alpha^{k}$. But to model second-period behavior, we must do so. At the beginning of the second period, each agent $k$ owns the endowments $e_{c_{2}}^{k}$ of second-period consumption goods and $e_{f_{2}}^{k}+\alpha^{k}$ of factors. Using the sequential-trading-equilibrium values of $p_{2}$ and $w_{2}$, agent $k$ 's budget constraints imply that $\alpha^{k}$ must satisfy

5 Since there are two budget constraints for each agent in the sequential model, an uninteresting nominal multiplicity of equilibria appears that is absent from the intertemporal model: given sequential-trading-equilibrium prices $\left(p_{1}, p_{2}, w_{2}, R\right)$ and a scalar $\lambda>0$, $\left(p_{1}, \lambda p_{2}, \lambda w_{2}, \lambda R\right)$ are also sequential-trading-equilibrium prices. The multiplicity only involves a rescaling of second-period prices, however, and so allocations of goods do not change. 


$$
p_{2} \cdot z_{2}^{k}\left(p_{1}, p_{2}, w_{2}, R\right)=w_{2} \cdot\left(\alpha^{k}+e_{f_{2}}^{k}\right) .
$$

And in the aggregate, total portfolio holdings of the produced factors must equal total production of factors:

$$
\sum_{k} \alpha^{k}=A_{31} y_{1} .
$$

Unfortunately, unless there is either a single agent or a single produced factor, (5.3) and (5.4) do not determine a single set of values for the $\alpha^{k}$. Typically, therefore, a sequential trading equilibrium will generate a multi-dimensional set of second-period economies, each of which can be identified with one set of values for the $\alpha^{k}$. The set of second-period economies that correspond to a sequential trading equilibrium can therefore be viewed as a finite-dimensional Euclidean space.

Once we stipulate the $\alpha^{k}$, all of the primitives of a standard Arrow-Debreu economy are specified. The technology matrix is given by the second-period activities of the original intertemporal economy's technology matrix, endowments are as defined above, and each $k$ maximizes the utility function $u^{k}\left(x_{1}^{k}, x_{2}^{k}\right)$ subject to $p_{2} \cdot x_{2}^{k} \leq p_{2} \cdot e_{c_{2}}^{k}+w_{2} \cdot\left(\alpha^{k}+e_{f_{2}}^{k}\right)$, where $x_{1}^{k}=z_{1}^{k}\left(p_{1}, p_{2}, w_{2}, R\right)+e_{c_{1}}^{k}$ is now an exogenous variable determined by the first period of the sequential trading equilibrium. We will say that an intertemporal equilibrium induces this second-period economy.

Letting $z_{2}\left(p_{2}, w_{2}\right)$ denote the aggregate excess demand function for the second-period economy (a function that is different from the $z_{2}$ used to model the intertemporal economy), a second-period equilibrium is a $\left(p_{2}, w_{2}, y_{2}\right)$ that satisfies $z_{2}\left(p_{2}, w_{2}\right) \leq A_{22} y_{2},-A_{32} y_{2} \leq A_{31} y_{1}+$ $e_{f_{2}}$, and $p_{2}{ }^{\prime} A_{22}+w_{2}{ }^{\prime} A_{32} \leq 0$, where $y_{1}$ is fixed at its sequential-trading-equilibrium value.

\section{Sequential indeterminacy}

Given a sequential trading equilibrium $\left(p_{1}, p_{2}, w_{2}, R, y_{1}, y_{2}\right)$, it is easy to confirm that $\left(p_{2}, w_{2}, y_{2}\right)$ is an equilibrium, which we call the continuation equilibrium, of any of the induced 
second-period economies. At the continuation equilibrium, the expectations of second-period prices anticipated in the sequential trading equilibrium are fulfilled. But are continuation equilibria determinate? When, for each intertemporal economy in some nonempty open set, there exists an equilibrium where the continuation equilibrium of almost all of the induced second-period economies lies amid a continuum of second-period equilibria, we say that sequential indeterminacy occurs.

Analogously to the original intertemporal model, we use bars to indicate that factors in excess supply and activities that earn negative profits (or that utilize or produce only factors in excess supply) at the continuation equilibrium have been omitted from $w_{2}, e_{f_{2}}, y_{1}, y_{2}, A_{22}, A_{31}$, and $A_{32}$, that the first coordinate of $p_{2}$ has been set to one, and that the first coordinate of $z_{2}$ has been omitted. (As in section 2, Walras' law allows us to ignore one market-clearing condition, which we choose to be the first of the second-period consumption goods.) The matrix $\tilde{A}_{22}$ omits the row of $\bar{A}_{22}$ that corresponds to the first of the second-period consumption goods. Finally let $\bar{M}_{2}$ indicate the number of factors where demand exactly equals supply at the continuation equilibrium and $\bar{N}_{2}$ denote the number of activities in use at the continuation equilibrium. Equilibria are then locally characterized by the equations:

$$
\begin{aligned}
& \bar{z}_{2}\left(\bar{p}_{2}, \bar{w}_{2}\right)=\tilde{A}_{22} \bar{y}_{2}, \\
& -\bar{A}_{32} \bar{y}_{2}=\bar{A}_{31} \bar{y}_{1}+\bar{e}_{f_{2}}, \\
& \bar{p}_{2}^{\prime} \bar{A}_{22}+\bar{w}_{2}^{\prime} \bar{A}_{32}=0 .
\end{aligned}
$$

Conditions (6.1) to (6.3) consist of $L_{2}-1+\bar{M}_{2}+\bar{N}_{2}$ equations in the same number of unknowns the variables, $\bar{p}_{2}, \bar{w}_{2}$, and $\bar{y}_{2}$.

Recall from the example of section 3 that indeterminacy occurs at equilibria where a single activity fully employs two inelastically supplied factors. The example generalizes to cases where $m$ positively priced (and hence fully employed) factors are used by $n<m$ activities. Mimicking the argument from the example, suppose we fix the pertinent $n$ coordinates of $\bar{y}_{2}$ at 
their continuation equilibrium values. Independently of the value of the other $L_{2}-1+\bar{M}_{2}+$ $\bar{N}_{2}-n$ endogenous variables, the market-clearing conditions for the $m$ factors will remain satisfied. The remaining equalities therefore constitute a system of $L_{2}-1+\bar{M}_{2}+\bar{N}_{2}-m$ equations in $L_{2}-1+\bar{M}_{2}+\bar{N}_{2}-n$ variables: the difference between the number of variables and equations is $m-n>0$. This excess of variables over equilibrium conditions suggests that the system is indeterminate. To reach this conclusion formally, via the implicit function theorem, a rank condition must be satisfied. (That is, the derivatives of the remaining equalities with respect to the remaining endogenous variables must be a matrix of rank $L_{2}-1+\bar{M}_{2}+\bar{N}_{2}-m$.)

Of course, for almost every value of the right hand side of (6.2), there will be no set of $m$ fully employed factors that are used by $n<m$ activities: the $m$ equalities in (6.2) that correspond to the factors in question would have fewer than $m$ endogenous variables (the $n$ relevant coordinates of $y_{2}$ ). But consider again the intertemporal equilibrium that generates the secondperiod economy. From this perspective, (6.2) is also an equilibrium condition, but the additional variable $\bar{y}_{1}$ is endogenous. In the intertemporal equilibrium, therefore, the same $m$ equalities may well have $m$ or more endogenous variables. Hence, these $m$ equalities can robustly have a solution. In short, from the vantage point of the intertemporal equilibrium, the $m$ second-period factors can be used by or produced by at least $m$ activities in the two time periods taken together, and hence the intertemporal equilibrium can be regular, but, from the vantage point of the second-period equilibrium, the factors may well be used by fewer than $m$ second-period activities, and hence the continuation equilibrium can be indeterminate.

Summarizing the discussion so far, we conclude that if a regular intertemporal equilibrium has a set of $m$ positively priced second-period factors used by fewer than $m$ secondperiod activities and if the rank condition mentioned above is satisfied, then a robust case of sequential indeterminacy occurs. Curiously, the regularity of the intertemporal equilibrium is key to establishing robustness: it implies that the same factors continue to be fully employed and 
have positive prices and the same activities continue to be in use as the parameters of the overall two-period model change slightly.

To see a simple example of how second-period indeterminacy arises, suppose there is one consumption good per period and two factors in the second period. The first-period consumption good may either be consumed directly or used as the sole input in an intertemporal activity that produces the first of the second-period factors. The two second-period factors are then used as inputs in a single activity that produces the second-period consumption good. If, at some intertemporal equilibrium, both of the second-period factors are fully employed, (6.2) takes the form

$$
\begin{aligned}
& -A_{32}(1) y_{2}=A_{31}(1) y_{1}+e_{f_{2}}(1) \\
& -A_{32}(2) y_{2}=e_{f_{2}}(2),
\end{aligned}
$$

where $A_{32}(i), A_{31}(1)$, and $e_{f_{2}}(i)$ denote, respectively, the quantity of factor $i$ used by the secondperiod activity, the quantity of factor 1 produced by the intertemporal activity, and the aggregate endowment of factor $i$. Independently of $e_{f_{2}}$, these two equations always have a solution $\left(y_{1}, y_{2}\right)$ (and can robustly have a positive solution) if $A_{32}(2)$ and $A_{31}(1)$ are nonzero. But during the second period, when $y_{1}$ is fixed, the two fully employed factors are used by only a single activity. We are thus in the indeterminate case discussed in section 3. The isoquants in the second period are L-shaped; when both second-period factors are fully employed, the second-period factor endowments $-\left(A_{31}(1) y_{1}+e_{f_{2}}(1), e_{f_{2}}(2)\right)-$ lie exactly at an isoquant vertex, analogously to the fact that the indeterminacy endowments in Figure 1 lie on along the activity rays. Just as in that case, the marginal products of second-period factors are not well defined.

If there are multiple activities in the second period, the production possibilities frontier (PPF) - the boundary of the set of feasible aggregate first- and second-period consumptions appears as in Figure 2. The segments in the PPF with a strictly negative slope (excluding the kinks) occur when two second-period activities are in use (except possibly in the rightmost 
negatively sloped segment, where it could be that one activity is in use and the nonproduced factor is in excess supply): as each additional unit of first-period consumption is sacrificed, the mixture of second-period activity levels will shift slightly towards whichever activity uses the produced input more intensively (or more of the nonproduced factor is drawn into production). The kinks between the negatively sloped segments occur at the switch points when only one second-period activity is in use and thus correspond to second-period indeterminacy.

\section{[Insert Figure 2]}

If the model contains only a single agent (or many agents with identical homothetic preferences), the intertemporal equilibrium can be represented by adding indifference curves between first- and second-period consumption to Figure 2. Equilibrium is determined by a tangency between an indifference curve and the PPF. Evidently, tangencies can occur robustly at the kinks in Figure 2; if an equilibrium at a kink is regular, small changes in the two-period economy's parameters do not shift the equilibrium to one of the flat segments of PPF. Tangencies at the flat segments are equally robust. Thus, both sequential indeterminacy and regularity are normal events.

Sequential indeterminacy is easy to analyze when there is only one second-period consumption good (as in the above example): indeterminacy then necessarily occurs if some set of $m$ fully employed factors is used by fewer than $m$ activities. Due to the Walras' law omission of the market-clearing condition for the consumption good, (6.1) consists of no equations in this case. With multiple second-period consumption goods, we must use the implicit function theorem to establish indeterminacy. In exceptional cases, the rank condition mentioned earlier may not be satisfied and a formal proof of indeterminacy will not go through. Fortunately, the rank condition is satisfied for almost every induced second-period economy. Define an intertemporal equilibrium to be potentially degenerate if there is a set of $m$ positively priced second-period factors used by fewer than $m$ activities. A slight variant of the following theorem 
is proved in Mandler (1995).

Sequential Indeterminacy Theorem. There is a generic set of economies such that (1) if an intertemporal equilibrium is potentially degenerate then the continuation equilibrium of almost every induced second-period economy is indeterminate, and (2) if an intertemporal equilibrium is not potentially degenerate then the continuation equilibrium of almost every induced secondperiod economy is regular.

Thus, generically, all that matters for second-period determinacy is whether or not the number of second-period activities using each set of positively priced second-period factors is at least as large as the number of factors in that set.

7. The significance of sequential indeterminacy

By itself, the number of equilibria is a relatively narrow concern. The disturbing implication of sequential indeterminacy is that the second-period equilibria need not vary continuously as a function of the second-period parameters. Consider the case again when there are two factors, one activity, and one consumption good in the second period, and suppose the continuation equilibrium prices $(w(1), w(2))$ are strictly positive. If any quantity of factor $i$ is withdrawn from the market, the other factor will be in excess supply, sending its price to 0 , and raising $w(i)$ to $1 / a_{1 j}$. Consequently, no matter how small agents are as a proportion of the market, it is their interest to manipulate their factor supplies. If there is more than one secondperiod activity, the jump in factor prices need not be as large, but the discontinuity will remain, and agents will still have an incentive to manipulate market prices.

Price-taking behavior consequently becomes implausible, and some noncompetitive mechanism must pin down factor incomes. As an example, suppose factor incomes in the second 
period are set by Nash bargaining among factor owners. Investors in capital goods would then anticipate that their returns will deviate from competitive levels and thus would supply suboptimal quantities of capital goods. The institutional response to sequential indeterminacy can thus induce a hold-up problem.

Even if factor markets in the second period do operate competitively when the equilibrium is indeterminate, it is difficult to see how a perfect-foresight equilibrium would proceed through time: there is no mechanism to lead second-period markets to equilibrate at the continuation equilibrium prices. The continuation equilibrium is just one element of a continuum of equilibria; only the expectations that agents formed during the first period, and not any feature of markets narrowly construed, distinguish the continuation equilibrium from the rest. Moreover, since agents will foresee this difficulty in the first period, they will not anticipate any price vector with certainty; even in the first period, therefore, behavior will differ from competitive equilibrium predictions.

Intertemporal models with linear activities thus cannot operate via anonymous competitive markets. In order to replicate the competitive outcome, factor owners would have to sign long-term contracts that commit them to trade factors at prearranged prices. Although it is by no means unusual, nowadays, for factor markets to be modeled as long-term contracts, most analyses attribute the need for contracts to information asymmetries or to bilateral monopoly. The present account ascribes contracts to the very nature of technology and markets.

\section{What drives sequential indeterminacy?}

Three features of an intertemporal economy are crucial for sequential indeterminacy.

First, technology must be modeled as a discrete set of activities, not as a continuum of techniques. Second, a long-run or steady-state equilibrium concept must not be in place. Third, at least some factors must be inelastically supplied. 
We discuss the first two points in sections 9 and 11 below. As for inelastic factor supply, it should already be clear that our indeterminacy arguments hinge on factor supply being unresponsive to price changes. Observe though that we could allow factor supply to be locally, but not globally, inelastic. As long as price changes in the neighborhood of a continuation equilibrium do not induce a factor supply response, our earlier sequential indeterminacy arguments apply unaltered.

9. Choice of technique and the neoclassical production function

The neoclassical production function, differentiable at all points in its domain, implies that factors always have well-defined marginal products. Even the smallest change in factor prices then induces a change in factor demand, and consequently robust cases of sequential indeterminacy cannot occur.

Consider a constant-returns-to-scale production set $Y=\left\{x \in R^{n}: g(x) \leq 0\right\}$ that gives the aggregate production possibilities available for some set of $n$ technologically related goods. The elements of $Y$ use the standard sign convention for outputs and inputs, and we assume that $g$ is convex and differentiable and that $D g(x) \gg 0$ for all $x$. In equilibrium, producers will choose aggregate quantities $x$ so as to maximize $\bar{p} \cdot x$ subject to $g(x) \leq 0$, where $\bar{p}=(1, p(2), \ldots, p(n))$ is a normalized price vector. Define $\bar{p}$ to be normalized equilibrium prices for $x$ if $x$ solves this maximization problem at prices $\bar{p}$. At a full competitive equilibrium, analogous profitmaximization conditions must be satisfied for other technologically related sets of goods and markets must clear.

Suppose, in the manner of our earlier indeterminacy arguments, that we fix $x$ and that both $\bar{p}$ and $\bar{p}^{*}$ are normalized equilibrium prices for $x$. The Kuhn-Tucker theorem implies that there exist $\lambda>0$ and $\lambda^{*}>0$ such that $\bar{p}=\lambda D g(x)$ and $\bar{p}^{*}=\lambda^{*} D g(x)$. Hence, $(1 / \lambda) \bar{p}=$ $\left(1 / \lambda^{*}\right) \bar{p}^{*}$. Since $\bar{p}(1)=\bar{p}^{*}(1), \lambda=\lambda^{*}$ and $\bar{p}=\bar{p}^{*}$. Thus, there can be only one vector of 
normalized equilibrium prices for any set of aggregate quantities.

This reasoning does not show that competitive equilibria with differentiable technologies are locally unique - not even if $Y$ were the aggregate production set for the economy as a whole. Local uniqueness of a competitive equilibrium with prices $\bar{p}$ and aggregate quantities $x$ requires in addition that there are no other competitive equilibria with prices $\bar{p}^{\prime}$ and quantities $x^{\prime} \neq x$ that are arbitrarily close to $(\bar{p}, x)$. The standard regularity literature assures us that this property holds generically. To be convincing, however, a generic local uniqueness theorem must be set in an intertemporal framework and should not rely on arbitrary endowment perturbations. See Mandler (1997) for a proof of sequential determinacy - the determinacy of both the intertemporal equilibria and the second-period equilibria they endogenously generate - when technology is differentiable.

10. Sraffa and sequential indeterminacy ${ }^{6}$

Several parallels between sequential indeterminacy and Sraffa's indeterminacy argument in Production of Commodities by Means of Commodities have already surfaced. First, sequential indeterminacy occurs under linear activities but not with differentiable "marginal productivity" technologies. Second, Sraffa fixes the aggregate quantities produced and finds multiple equilibrium prices for those quantities. Our indeterminacy arguments follow the same method: we fix a subset of quantities and show that a continuum of price vectors support those quantities. Third, in line with Sraffa's emphasis on the production of commodities by means of commodities, it is only in dynamic models that indeterminacy arises systematically.

Sequential indeterminacy also sheds light on some of Sraffa's more elusive remarks. Sraffa was critical of theories that treat the economy as a "one-way avenue that leads from

\footnotetext{
6 This subject is treated more thoroughly in Mandler (1999b).
} 
'factors of production' to 'consumption goods.'” From the vantage point of sequential indeterminacy, the difficulty with "one-way" theories is not that they assume that final output is limited by society's factor resources, but that they let endowments be arbitrary rather than determined by ongoing investment decisions. Taking endowments to be in an arbitrary or generic set ensures that marginal products are well-defined even when technology is described by linear activities. (Recall from Figure 1 that marginal products are defined at and only at the generic endowment points at which factor prices are determinate.) Given that the endowments at which marginal products are ill-defined arise systematically in a sequential setting, Sraffa's suspicion of the device of an arbitrary starting point to economic activity accurately points to a serious difficulty in the foundations of marginal productivity theory.

But there are also clear differences between Sraffa's position and sequential indeterminacy. Our emphasis on inelastic factor supply has no parallel in Sraffa or the subsequent literature. More significantly, we will see sequential indeterminacy does not arise when equilibria are long-run.

Consider the following variant of Sraffa's model. Suppose $n$ material inputs and labor produce the same $n$ material goods, with the outputs appearing one period after the inputs are applied. Each commodity $j$ is produced by one linear activity, represented by an $n$-vector $a_{j}=$ $\left(a_{1 j}, \ldots, a_{n j}\right) \geq 0$ of material input requirements and a scalar $\ell_{j} \geq 0$ of labor required. Capital invested in each activity earns the same rate of profit $r$. Assuming as Sraffa does that labor is purchased when output is sold, profit rate equalization implies, for each produced good $j$, that

$$
p(j)=(1+r)\left(p(1) a_{1 j}+\ldots+p(n) a_{n j}\right)+w \ell_{j},
$$

where $p(j)$ is the price of $\operatorname{good} j$, and $w$ is the wage. Letting $A=\left[a_{1} \cdots a_{n}\right], \ell=\left(\ell_{1}, \ldots, \ell_{n}\right)$, and normalizing by defining $\bar{p}=(1, p(2), \ldots, p(n))$, we can rewrite the above equations as

$$
\bar{p}=(1+r) A^{\prime} \bar{p}+w \ell .
$$

Since (10.1) constitutes $n$ equations in the $n+1$ unknowns $(\bar{p}, w, r)$, the equations have one 
degree of freedom. Hence, one variable can be varied exogenously while still allowing (10.1) to be satisfied. Sraffa lets $r$ be this exogenous variable and concludes that competition leaves the interest or profit rate indeterminate.

Neoclassical critics, most thoroughly Hahn (1982), have faulted Sraffa's model on two main counts. First, Sraffa omits any mention of demand and supply. Possibly, therefore, some of the price vectors that solve (10.1) might be inconsistent with market-clearing. Second, input and output prices are constrained to be equal in Sraffa's model: (10.1) uses the same $\bar{p}$ on both left and right hand sides. According to the critics, a proper equilibrium model should allow prices to change through time. Indeed, except at particular combinations of endowments and preferences, equilibria will not exist if prices across time periods are required to be equal.

The literature on Sraffa presumes that once these two flaws are rectified Sraffa's model necessarily becomes determinate. Our earlier indeterminacy results suggest, however, that determinacy is not guaranteed. Notice that the two objections to Sraffa work in opposite directions. Including market-clearing equations diminishes the potential for indeterminacy, but distinguishing prices by date adds new price variables and makes indeterminacy more likely. These two corrections turn out to offset each other exactly.

To meet the neoclassical objections, we distinguish between input prices $\bar{p}^{1}$ and output prices $\bar{p}^{2}$, and posit an aggregate demand function for the $n$ outputs $x\left(\bar{p}^{1}, \bar{p}^{2}, w, r\right)$. We assume that the $n$ material inputs are inelastically supplied at the level $e \gg 0$ and that labor is inelastically supplied at the level $e_{\ell}>0 .^{7}$ An equilibrium is a $\left(\bar{p}^{1}, \bar{p}^{2}, w, r, y\right)$ such that

$$
\begin{aligned}
& x\left(\bar{p}^{1}, \bar{p}^{2}, w, r\right) \leq y, \\
& \bar{p}^{2} \leq(1+r) A^{\prime} \bar{p}^{1}+w \ell, \\
& A y \leq e,
\end{aligned}
$$

7 We require that the model obeys Walras' law: for all $\left(\bar{p}^{1}, \bar{p}^{2}, w, r\right)$, $\bar{p}^{2} \cdot x\left(\bar{p}^{1}, \bar{p}^{2}, w, r\right)=(1+r) \bar{p}^{1} \cdot e+w e_{\ell}$. 


$$
\ell \cdot y \leq e_{\ell} .
$$

We suppose that conditions (10.2) to (10.5) hold with equality.

We now apply the indeterminacy arguments of section 6 . At an equilibrium $\left(\bar{p}^{1}, \bar{p}^{2}, w, r, y\right)$ where each price, activity level, and $(1+r)$ is strictly positive, the total number of factors with positive prices $(n+1)$ will be larger than the number of activities in use $(n)$. Assuming the appropriate rank condition is satisfied, the arguments of section 6 imply that the equilibrium must be indeterminate. It is just as easy to reason directly. If we fix $y$ at an equilibrium value, (10.4) and (10.5) remain satisfied at all values of $\left(\bar{p}^{1}, \bar{p}^{2}, w, r\right)$. The remaining equations consist of the $n-1$ independent equations in (10.2) - we lose an equation due to Walras' law - and the $n$ equations in (10.3). With $y$ fixed, the endogenous variables consist of only the $2 n$ prices $\left(\bar{p}^{1}, \bar{p}^{2}, w, r\right)$. With one more unknown that equation, indeterminacy typically obtains.

Interestingly, the dimension of sequential indeterminacy tracks the dimension of indeterminacy in Sraffa's theory. Suppose, in the second-period model of section 6, that each factor is fully employed and has a positive price and that the indeterminacy-inducing set of factors is the economy's entire set of second-period factors. The dimension of indeterminacy then equals $M_{2}$, the total number of second-period factors, minus $\bar{N}_{2}$, the number of activities in use. Hence, multi-dimensional indeterminacy - indeterminacy of dimension greater than $1-$ occurs when $M_{2}-1>\bar{N}_{2}$. In Sraffa's model of joint production, where activities can produce more than one output, the number of activities in use can fall below $n$ even while all $n$ goods are produced. Sraffa recognized that in this case the dimension of indeterminacy can expand beyond the single dimension claimed for (10.1). In Sraffa's words, multidimensional indeterminacy obtains when there are "more [goods] prices to be ascertained than there are processes" in use. Since the number of goods prices, $n$, equals the total number of factor prices (the material inputs plus labor) minus 1, Sraffa's condition for multidimensional indeterminacy is the same as ours. 
Curiously, although he recognized the possibility of multidimensional indeterminacy, Sraffa assumed it away by supposing that the number of activities in use always equals the number of goods. Also, Sraffa failed to report that indeterminacy can disappear when the number of activities in use is greater than $n$.

We have departed markedly from Sraffa in not making the "long-run" assumption that relative prices remain constant through time. If relative prices are to remain constant, the economy must be placed in an infinite-horizon setting. However, while sequential indeterminacy can easily arise in an infinite-horizon model, the long-run equilibria where relative prices are required to be constant are determinate. We sketch a simple model of the long run to establish this point. More details can be found in Mandler (1999b). The key additional ingredient is a steady state demand function, $x(\bar{p}, w, r)$, which we let originate from overlapping generations of agents. The $n$ goods are partitioned into a set of consumption goods and pure factors, and the subscripts $c$ and $f$ respectively denote the rows of the input requirement matrix and the entries of $y$ that correspond to these goods. Assuming for simplicity that all equilibrium conditions hold with equality, a long-run equilibrium is a $(\bar{p}, w, r, y)$ such that

$$
\begin{aligned}
& x(\bar{p}, w, r)+A_{c} y=y_{c} \\
& A_{f} y=y_{f} \\
& \ell \cdot y=e_{\ell} \\
& \bar{p}=(1+r) A^{\prime} \bar{p}+w \ell .
\end{aligned}
$$

Since none of the market-clearing equations are redundant in a long-run OLG setting, we have $2 n+1$ independent equations in the $2 n+1$ variables $(\bar{p}, w, r, y){ }^{8}$

One might suspect that sequential indeterminacy could arise in this model, since, with no

${ }^{8}$ We have modeled equilibria as steady state. But if the endowment of labor, $e_{\ell}$, were to grow at some fixed rate through time (or if each input requirement were to diminish at the same fixed rate), the model could with minor notational adjustments describe a growing economy instead. 
restrictions on $A$, some subset of $m$ material pure factors and labor may well be used by fewer than $m$ activities. Moreover, the endowment of material inputs in any given period, $y_{f}$, is an endogenous variable; hence, it can robustly occur that the market-clearing conditions for these $m$ factors are satisfied with equality. Nevertheless, we have the following result from Mandler (1999b), which extends Kehoe and Levine's (1985) analysis of exchange economies to models of production.

Long-run Determinacy Theorem. There is a generic set of labor endowments, technology parameters, and demand functions such that each long-run equilibrium is locally unique.

The reason for the determinacy of the long-run model is not hard to grasp. Although in any given period, some set of $m$ factors may be used by fewer than $m$ activities, each factor's price as an input is constrained to equal the price of the same good currently in production. These additional equilibrium conditions are enough to ensure generic determinacy. More general models with more nonproduced pure factors in addition to labor are also generically determinate.

In sum, our results support neither side of the debate over Sraffa. If relative prices can vary through time, Sraffa had the right building blocks of a coherent indeterminacy argument. He identified the close link between linear activities and indeterminacy and rightly emphasized the significance of not imposing an arbitrary starting point for economic activity. But in a longrun setting of constant relative prices, the neoclassical case for determinacy is convincing. Each side to the Sraffa debate thus backs an equilibrium concept inconsistent with its determinacy claims. 


\section{Concluding remarks: indeterminacy and long-run equilibria}

The indeterminacy associated with inelastic factor supply and linear activities was well recognized in pre-World War II economic theory. Hicks (1932) and Robertson (1931), for example, acknowledged that factor prices in the short run may be indeterminate. They recognized that highly specialized forms of labor and capital might not allow any leeway for factor substitution, and hence factor demand might not respond to price changes. Although they did not dismiss the short-run problem, Hicks and Robertson argued that equilibria would at least be determinate in the long-run. That is, even if deviations in factor prices from their long-run equilibrium values do not immediately disturb any equilibrium condition, persistent deviations will ultimately be incompatible with market clearing.

Hicks and Robertson, and most other prewar economists, analyzed factor markets in terms of aggregate basic factors, land, labor, and capital. Intermediate inputs and ultimately final output as well were viewed as reducible to the quantities of basic factors needed to produce them, and competitive equilibria were understood in terms of the demand for and the supply of basic factors. A set of basic factor prices determines cost-minimizing prices for final output; given these output prices, consumers choose their final output demands and thus indirectly determine the demand for basic factor inputs. Full long-run equilibrium occurs where basic factor demand, calculated in this way, equals basic factor supply.

Hicks and Robertson argued that if the economy begins at a position of long-run equilibrium then a permanent shift in basic factor prices will eventually change the demand for basic factors. Even if given stocks of intermediate inputs must be used in fixed proportions, opportunities for factor substitution emerge as new intermediate inputs become available. For instance, if the wage were to fall permanently, labor-intensive intermediate inputs will eventually become cheaper and will therefore be adopted in more production processes, ultimately raising labor demand. In addition, a fall in the wage will lower the price of labor-intensive final outputs, 
raising demand for these outputs and thus again raising labor demand. See Mandler (1999a) for an explicit model.

This long-run case for determinacy is considerably more convincing than direct factor substitution arguments. Since the birth of marginal productivity theory in the 1890's, a steady stream of economists has balked at applying factor substitution to specialized intermediate inputs. Explaining the elasticity of factor demand as a consequence of the switch to distinct intermediate inputs is vastly more plausible. Of course, as Hicks or Robertson acknowledged, at any given point in time, current-period prices of basic factors and intermediate inputs may still exhibit indeterminacy; only sustained changes in factor prices are likely to induce disequilibrium changes in factor demand.

Our determinacy and indeterminacy theorems bear out the prewar understanding of factor pricing. The determinacy of equilibria in the Hicks-Robertson sense finds formal expression in the Long-run Determinacy Theorem of section 10. And Hicks's and Robertson's claim that factor prices may nevertheless be indeterminate in the short-run is nothing more than an assertion of sequential indeterminacy. The only difference is that Hicks and Robertson did not dwell on changes in factor prices that persist for only a brief period of time; they shrugged off short-run indeterminacy and focused on the long run. But in both the Hicks-Robertson description of the short run and in the formal theory of sequential indeterminacy, the equilibrium that validates perfect-foresight expectations (long-term expectations in Hicks-Robertson) is surrounded by other equilibria.

The prewar approach to factor demand appears suspicious today in its cavalier aggregation of factors. In fact, the analytical difficulties that vex factor aggregation seem to have led Sraffa to believe that without aggregation, long-run models would be indeterminate. Certainly, Sraffa's most telling criticisms of neoclassical theory pertain to its aggregation claims. But as the Long-run Determinacy Theorem indicates, an indeterminacy indictment of the 
neoclassical theory of long-run equilibria cannot be sustained. Hicks and Robertson happened to reason in terms of aggregated basic factors, but the Long-run Determinacy Theorem (and its extensions) show that their analysis can be cleansed of any aggregation assumptions. Indeed, even the Hicks-Robertson confidence in long-run opportunities for factor substitution is unnecessary; the Long-run Determinacy Theorem relies only on the fact that long-run consumption prices (and hence consumption demand) change in response to shifts in long-run factor prices. Again, see Mandler (1999a) for details.

The determinacy of long-run equilibria hardly leaves factor price theory in satisfactory shape. As we mentioned, long-run equilibria suffer from the same sequential indeterminacy problems as do standard intertemporal equilibria; the only difference is that the equilibria near a long-run equilibrium do not have constant relative prices and therefore are not themselves longrun. And, just as with non-steady-state intertemporal equilibria, no pure market mechanism can force markets to equilibrate in each period at the long-run equilibrium prices and agents will have strong incentive to manipulate factor markets. Long-run equilibria thus also require an accompanying theory of factor markets contracts and contract enforcement.

\section{Bibliography}

G. Debreu, 1970, Economies with a finite set of equilibria, Econometrica 38: 387-93.

G. Fuchs, 1974, Private ownership economies with a finite number of equilibria, Journal of Mathematical Economics 1: 141-58.

F. Hahn, 1982, “The neo-Ricardians,” Cambridge Journal of Economics 6: 353-74.

J. R. Hicks, 1932, Marginal productivity and the principle of variation, Economica 25: 79-88.

T. Kehoe, 1980, An index theorem for general equilibrium models with production, Econometrica 48: 1211-32.

T. Kehoe, 1982, Regular production economies, Journal of Mathematical Economics 10: 147-76.

T. Kehoe and D. Levine, 1985, Comparative statics and perfect foresight in infinite horizon 
economies, Econometrica 53: 433-53.

T. Koopmans, 1995, Analysis of production as an efficient combination of activities, in "Activity Analysis of Production and Distribution," T. Koopmans, ed., Wiley, New York.

M. Mandler, 1995, Sequential indeterminacy in production economies, Journal of Economic Theory 66: 406-36.

M. Mandler, 1997, Sequential regularity in smooth production economies, Journal of Mathematical Economics 27: 487-504.

M. Mandler, 1999a, "Dilemmas in economic theory,” Oxford University Press, New York.

M. Mandler, 1999b, Sraffian indeterminacy in general equilibrium, Review of Economic Studies 66: 693-711.

A. Mas-Colell, 1975, On the continuity of equilibrium prices in constant returns production economies, Journal of Mathematical Economics 2: 21-33.

A. Mas-Colell, 1985, "The Theory of General Economic Equilibrium: A Differentiable Approach," Cambridge University Press, Cambridge.

R. Radner, 1972, Existence of equilibrium of plans, prices, and price expectations in a sequence of markets, Econometrica 40: 289-303.

K. Roberts, 1980, The limit points of monopolistic competition, Journal of Economic Theory 22: 256-279.

D. Robertson, 1931, Wage grumbles, in “Economic fragments," London, P.H. King.

S. Smale, 1974, Global analysis and economics, IV, Journal of Mathematical Economics 1: 119127.

P. Sraffa, 1960, "Production of Commodities by Means of Commodities," Cambridge:

Cambridge University Press. 


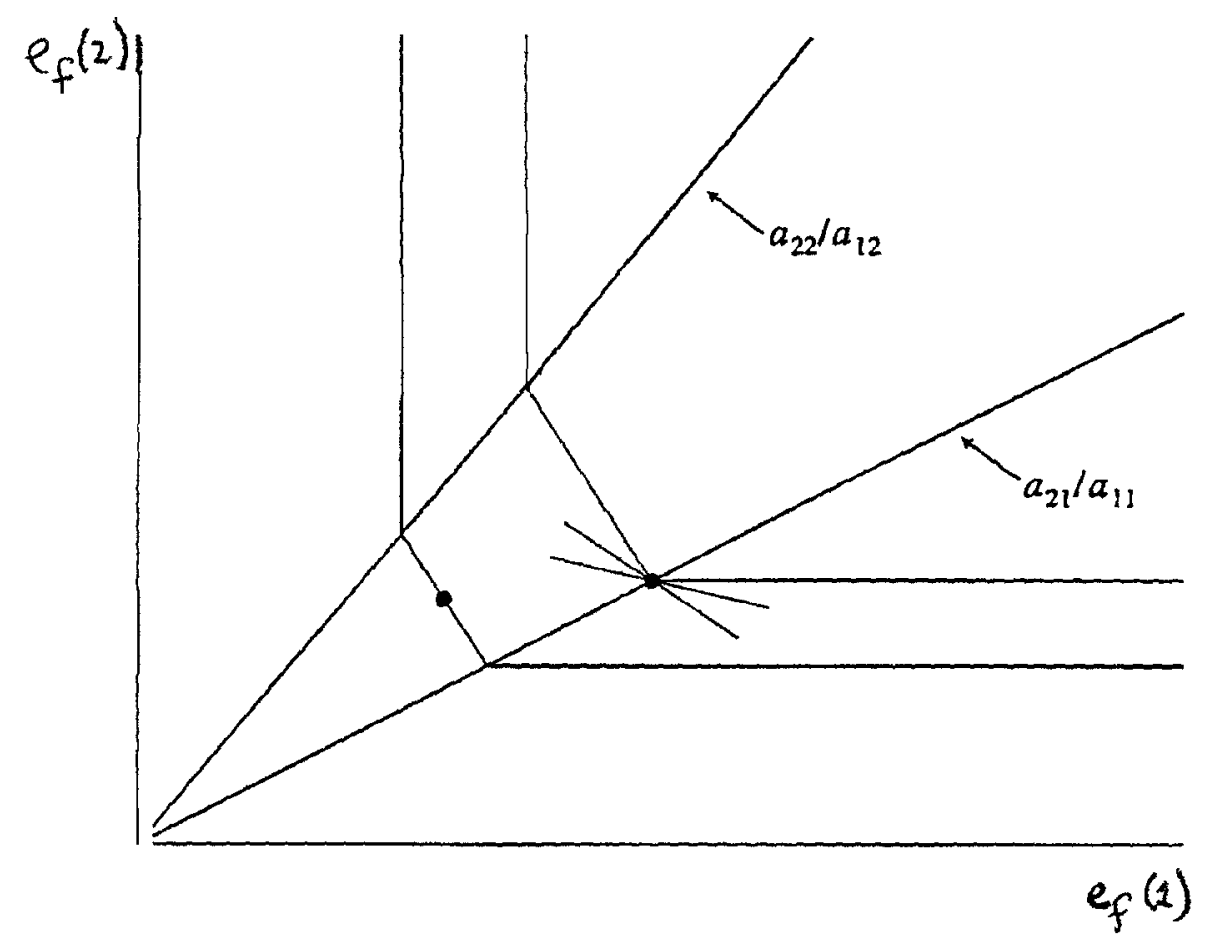

Figure 1 


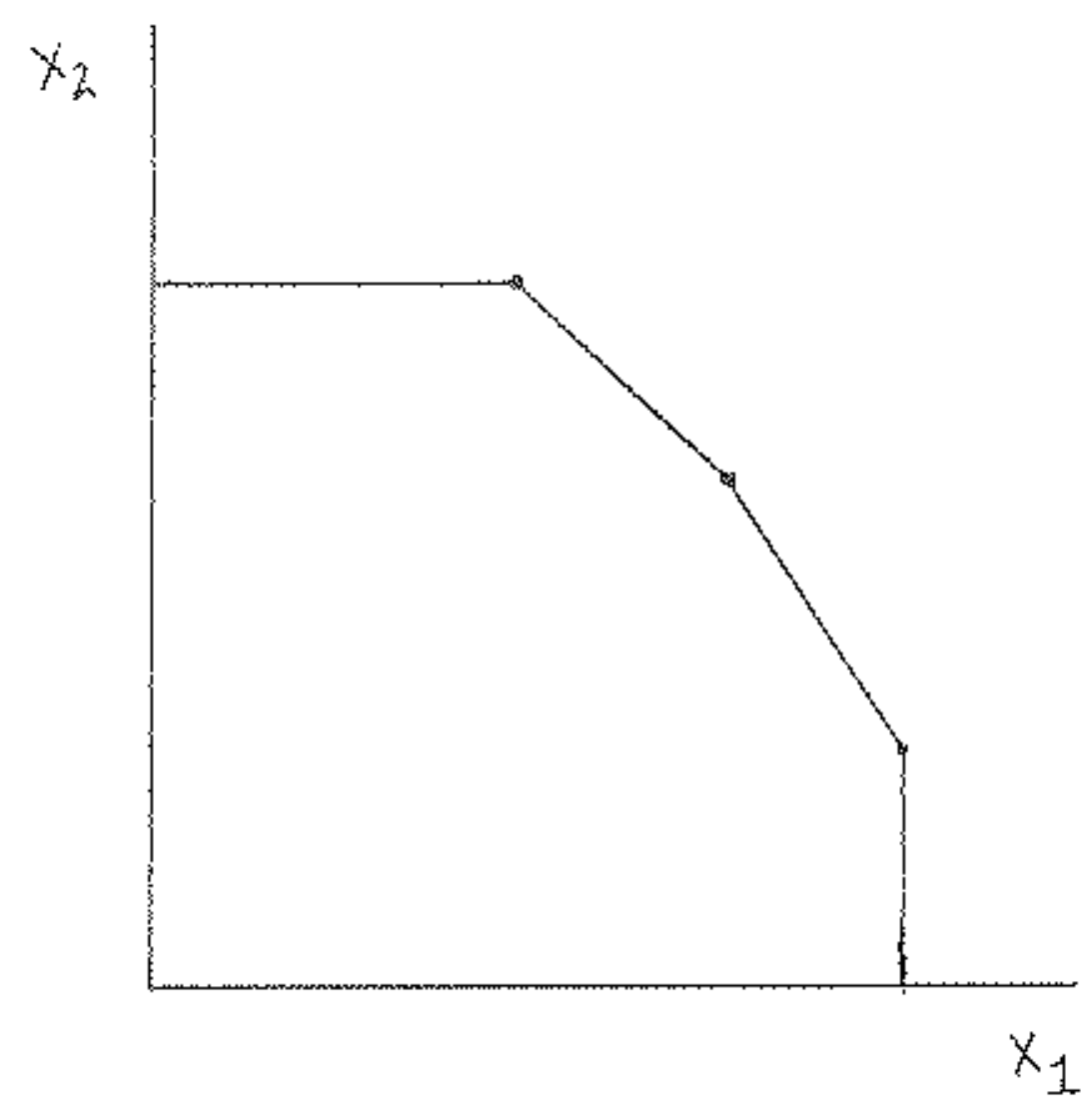

Figure 2 